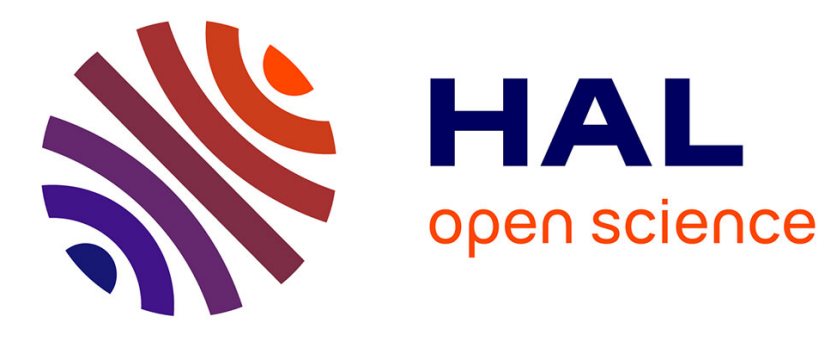

\title{
Three-dimensional stationary flow over a backward-facing step
}

Jean-François Beaudoin, Olivier Cadot, Jean-Luc Aider, Jose Eduardo

Wesfreid

\section{- To cite this version:}

Jean-François Beaudoin, Olivier Cadot, Jean-Luc Aider, Jose Eduardo Wesfreid. Three-dimensional stationary flow over a backward-facing step. European Journal of Mechanics - B/Fluids, 2004, 23 (1), pp.147-155. 10.1016/j.euromechflu.2003.09.010 . hal-00173133

\section{HAL Id: hal-00173133 \\ https://hal.science/hal-00173133}

Submitted on 2 Dec 2017

HAL is a multi-disciplinary open access archive for the deposit and dissemination of scientific research documents, whether they are published or not. The documents may come from teaching and research institutions in France or abroad, or from public or private research centers.
L'archive ouverte pluridisciplinaire HAL, est destinée au dépôt et à la diffusion de documents scientifiques de niveau recherche, publiés ou non, émanant des établissements d'enseignement et de recherche français ou étrangers, des laboratoires publics ou privés. 


\title{
Three-dimensional stationary flow over a backward-facing step
}

\author{
Jean-François Beaudoin ${ }^{\mathrm{a}, \mathrm{b}, *}$, Olivier Cadot ${ }^{\mathrm{a}}$, Jean-Luc Aider ${ }^{\mathrm{b}}$, \\ José Eduardo Wesfreid ${ }^{\text {a }}$ \\ a Physique et mécanique des milieux hétérogènes, École supérieure de physique et chimie industrielles de Paris \\ (PMMH UMR 7636-CNRS-ESPCI), 10, rue Vauquelin, 75231 Paris cedex 5, France \\ b PSA Peugeot Citroën, direction de la recherche, centre technique de Vélizy, route de Gisy, 78943 Vélizy-Villacoublay cedex, France
}

\begin{abstract}
Three-dimensional stationary structure of the flow over a backward-facing step is studied experimentally. Visualizations and Particle Image Velocimetry (PIV) measurements are investigated. It is shown that the recirculation length is periodically modulated in the spanwise direction with a well-defined wavelength. Visualizations also reveal the presence of longitudinal vortices. In order to understand the origin of this instability, a generalized Rayleigh discriminant is computed from a twodimensional numerical simulation of the basic flow in the same geometry. This study reveals that actually three regions of the two-dimensional flow are potentially unstable through the centrifugal instability. However both the experiment and the computation of a local Görtler number suggest that only one of these regions is unstable. It is localized in the vicinity of the reattached flow and outside the recirculation bubble.
\end{abstract}

\section{Introduction}

The phenomenon of flow separation is a problem of great importance for fundamental and industrial reasons. For instance it often corresponds to drastic losses in aerodynamic performances of airfoils or automotive vehicles. The backward-facing step is one of the simplest geometries to study this phenomenon. As a major benchmark for two-dimensional numerical simulations the backward-facing step has been the subject of experimental (see for instance Armaly et al. [1]) and numerical investigations (Kaiktsis et al. [2], Kaiktsis et al. [3], Kim and Moin [4], Lesieur et al. [5]).

Only a few studies are devoted to the three-dimensional aspects of this flow, especially in the steady regime. Armaly et al. [1] and Williams and Baker [6] focused on the extrinsic side-wall effects experimentally and numerically. More recently Barkley et al. [7] revealed with a linear stability analysis based on numerical simulations, that a steady three-dimensional bifurcation occurs at a critical Reynolds number (based on the step height and the maximum velocity of the upstream profile) of 748 . Their computation was performed on an infinite domain in the spanwise direction which suggests this instability to be intrinsic.

In the present article, the aim is also to give more insight about the origin of the three-dimensionality occurring in separated flows. We first describe the experimental set-up of the backward-facing step flow and the measurements. The results are then divided into two main parts. The first one concerns the experiment in which observations of the three-dimensional intrinsic instability are reported. Such observations, to our knowledge, do not have been reported before. In the second part we use numerical simulations of the two-dimensional basic flow in order to understand the three-dimensional instability. Experimental and numerical results are discussed together, which lead us to our conclusion concerning the possible mechanism responsible for the three-dimensional instability.

\footnotetext{
* Corresponding author.

E-mail address: beaudoin@ @mmh.espci.fr (J.-F. Beaudoin).
} 


\section{Experimental set up}

The flow is produced by gravity in a horizontal water tunnel. The rectangular cross section of the test channel is $150 \mathrm{~mm}$ wide and $100 \mathrm{~mm}$ high. Its total length is $820 \mathrm{~mm}$, which allows visualizations and measurements far downstream of the step (Fig. 1). The flow velocity ranges from 0.2 to $20 \mathrm{~cm} \cdot \mathrm{s}^{-1}$ with a precision of $0.05 \mathrm{~cm} \cdot \mathrm{s}^{-1}$. The step geometry is shown in Fig. 1; it is composed of a ramp of angle $9.5^{\circ}$ upstream of a backward-facing step of height $h$. For this geometry, the boundary layer does not separate except at the edge of the step. In our coordinates system (Fig. 1(a)) $x, y$ and $z$ are respectively the streamwise, vertical and spanwise directions. The origin $\mathrm{O}$ of this system is located in the plane of symmetry of the channel and in the step corner. The Reynolds number, $R e=h U^{0} / \nu$, is based on the step height $h$ and the maximum velocity of the step edge

(a)

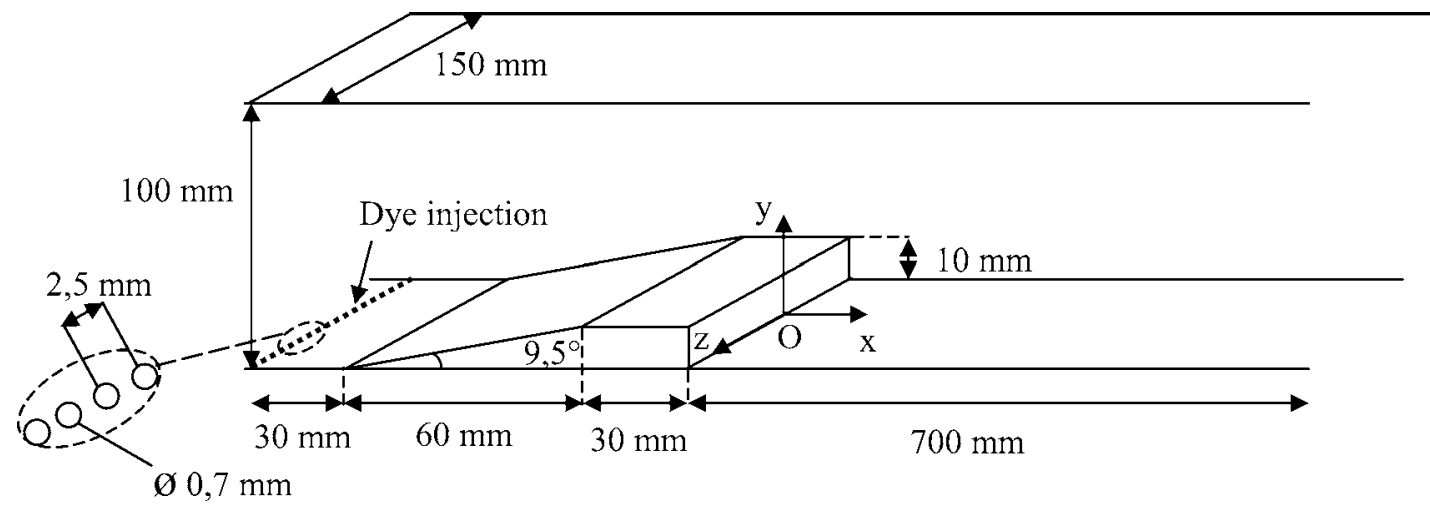

(b)

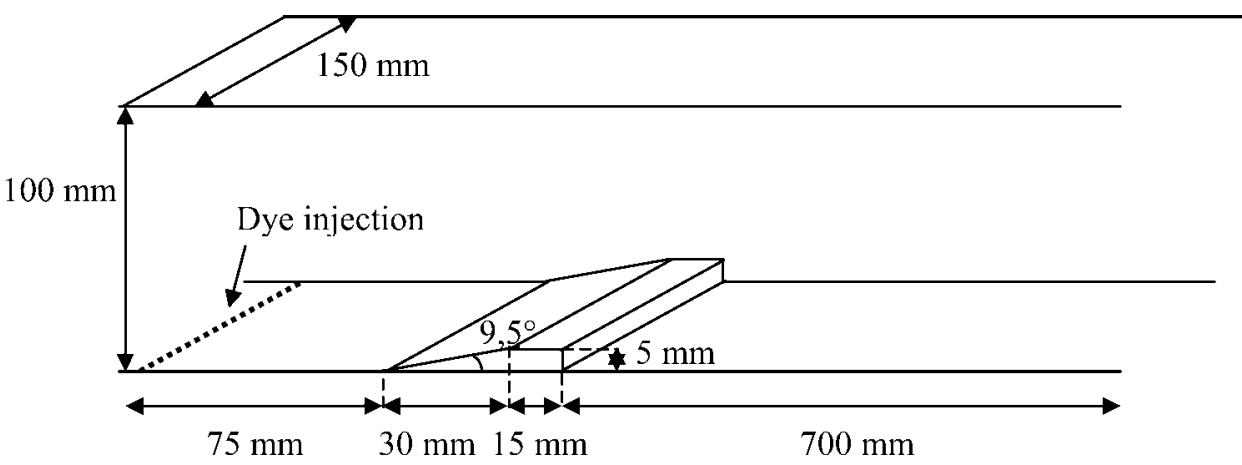

(c)

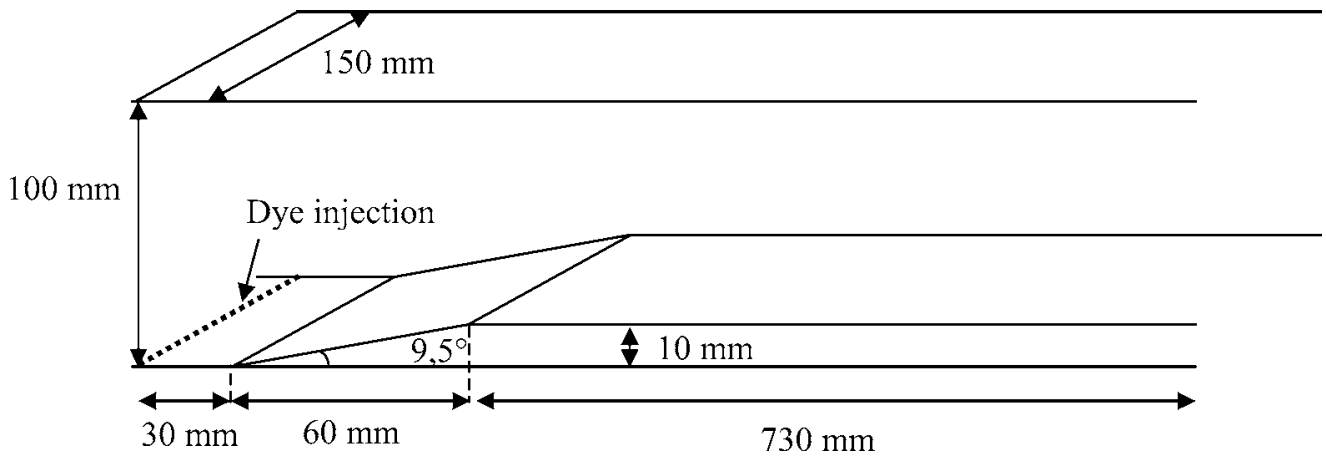

Fig. 1. Experimental set-up for the three configurations: (a) with the $10 \mathrm{~mm}$ high step; (b) with the $5 \mathrm{~mm}$ high step; (c) with the upstream ramp (no step). 


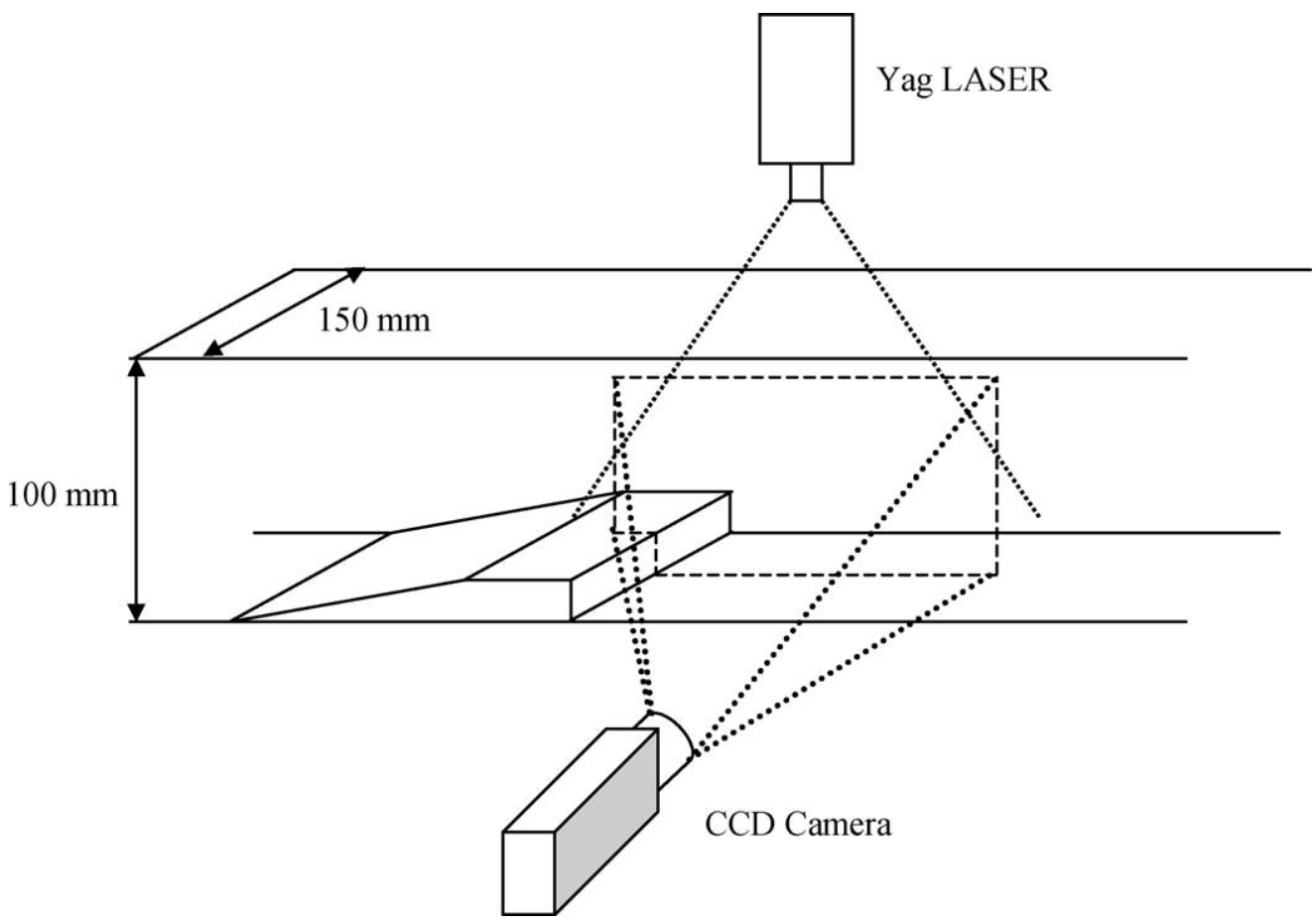

Fig. 2. Description of the PIV set-up. The measurements window is $75 \mathrm{~mm}$ high and $92 \mathrm{~mm}$ wide.

profile, $U_{0}$. With this definition, the Reynolds number ranges from 10 to 300 in the present study. Another definition is also often used for this geometry. It is based on the size of the inlet channel and the average upstream velocity [1-3]. We estimated the relation between the Reynolds number in our study and the Reynolds number in [1-3] by: $R e_{[1-3]}=\frac{4}{3} R e$.

We study three flow configurations. In the first one, the step height is $h=10 \mathrm{~mm}$ (Fig. 1(a)), and the expansion ratio: $H /(H-h)=1.11$. In the second configuration, the step height is $h=5 \mathrm{~mm}$ (Fig. 1(b)) and the expansion ratio: $H /(H-h)=1.05$. The third configuration is realized to quantify the influence of the upstream ramp with no step (Fig. 1(c)).

The flow is visualized by means of Laser Induced Fluorescence (LIF) in different planes $x=$ cst and $z=$ cst. The dye injection is performed in the upstream boundary layer through 50 holes of $0.7 \mathrm{~mm}$ in diameter (Fig. 1(a)). The apparatus used for the injection is similar to the one used by Cadot and Kumar [8]. The injection rate is simply imposed by the rotation frequency of a peristaltic pump, which allows a perfect control of the rate. A drawback of such a pump is that the dye is periodically pulsed due to the pinching of the flexible tubes. In order to smooth out the dye flux pulses, we insert between the pump and the injection holes a $250 \mathrm{ml}$ container partially filled with air: the free surface in the container removes the high-frequency pulsations. The dye injection velocity for each hole is $0.05 \mathrm{~cm} \cdot \mathrm{s}^{-1}$ and no influence on the flow was observed.

We use a standard Particle Image Velocimetry (PIV) set-up (Adrian [9]). The water is seeded with spherical particles, $11 \mu \mathrm{m}$ in nominal diameter. Two Nd:Yag laser sources with $12 \mathrm{~mJ}$ of energy per pulse each and a duration of 4 ns provide a doublepulsed light sheets. A $10 \mathrm{~mm}$ diameter cylindrical lens is used to expand the beam into a light sheet (Fig. 2) that is approximately $0.5 \mathrm{~mm}$ thick. Images are recorded using a $1280 \times 1024$ pixels CCD video camera. The physical dimensions of the PIV images in the $x-y$ plane is $75 \times 92 \mathrm{~mm}^{2}$. We use a $32 \times 32$ pixels interrogation window with a $50 \%$ overlap leading to $1.2 \mathrm{~mm}$ spatial resolution.

\section{Experimental results}

\subsection{Flow in the symmetry plane}

Fig. 3 shows both the visualization and the velocity profile in the symmetry plane $z=0$ of the $10 \mathrm{~mm}$ high step at $R e=100$. Because of the high aspect ratio of the channel, the velocity profile at the step edge is not a Poiseuille flow but a flat profile with about a $10 \mathrm{~mm}$ thick boundary-layer. In the recirculation zone, the velocities are very small compared to the velocity of the mean flow. The separation surface is then submitted to a strong shear. For higher Reynolds numbers, the flow becomes unsteady; 


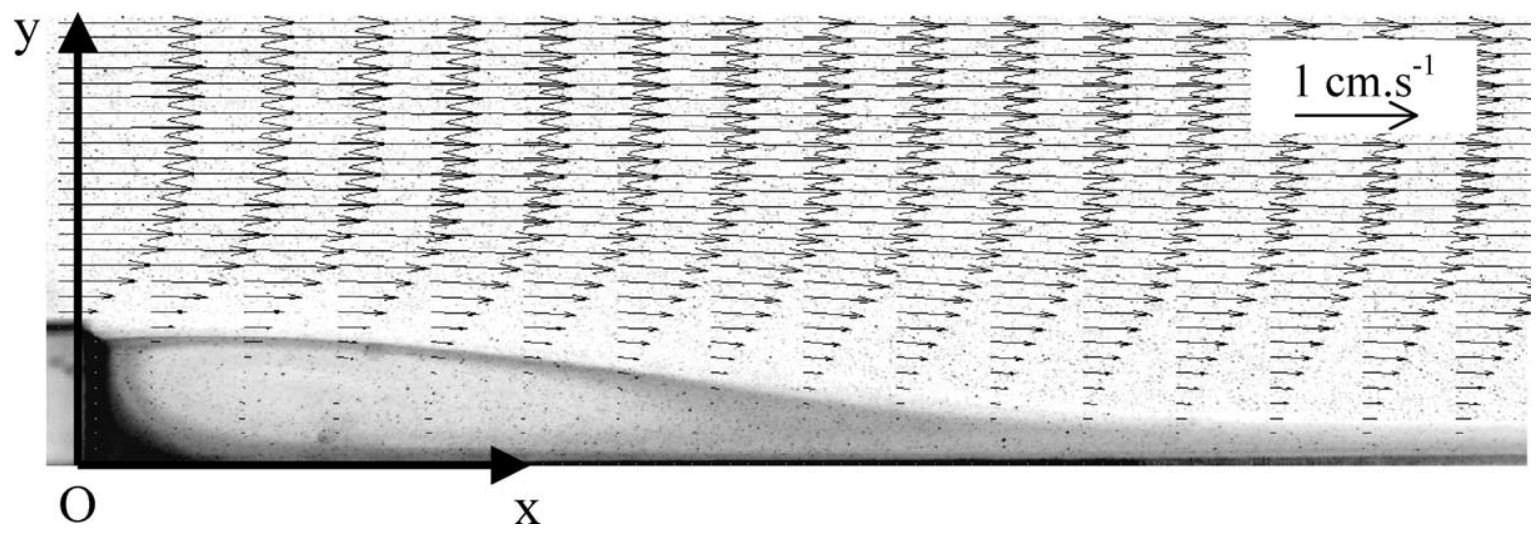

Fig. 3. Flow in the symmetry plane $z=0$ for $h=10 \mathrm{~mm}$ at $R e=100$ : visualization combined with PIV measurements.
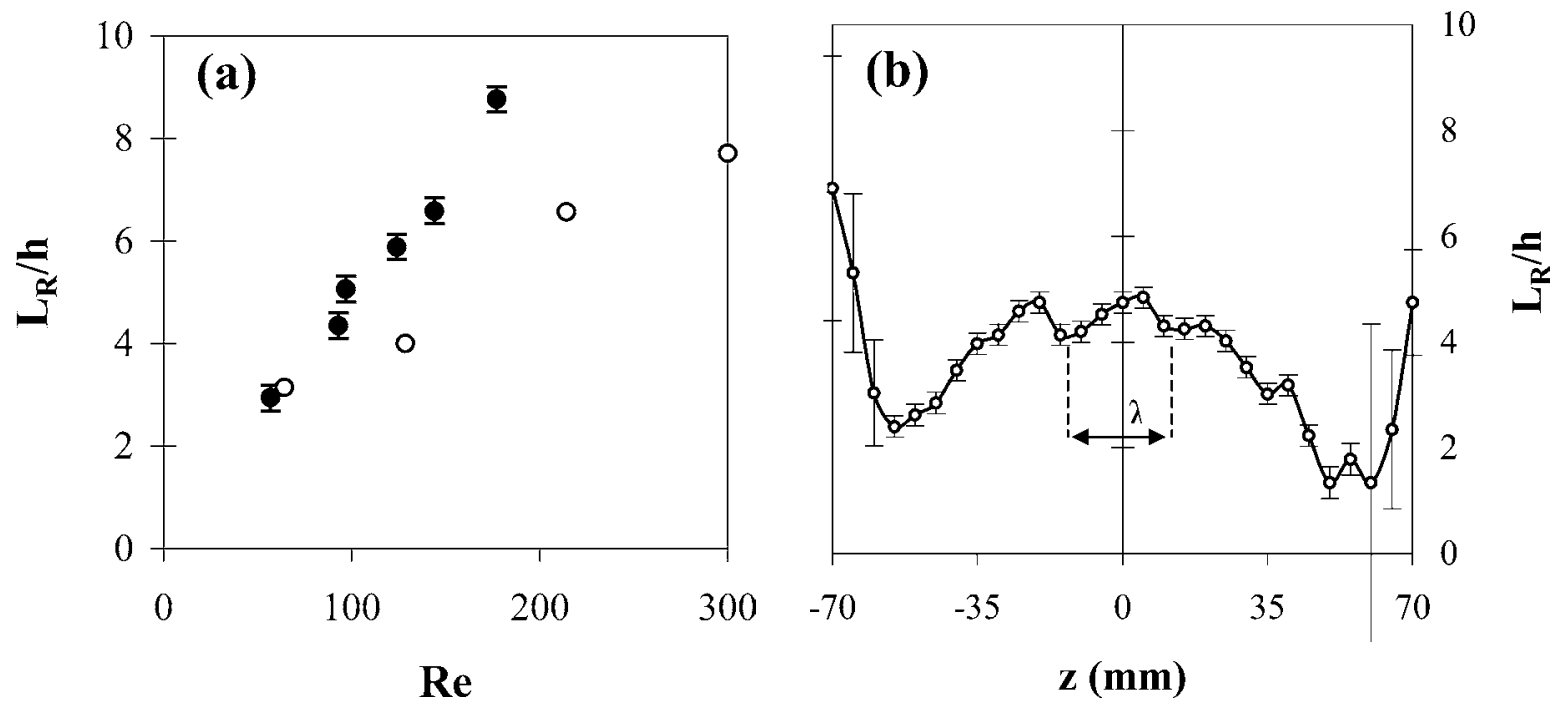

Fig. 4. Non-dimensional recirculation length for the $10 \mathrm{~mm}$ high step deduced from PIV measurements: (a) our measurements (filled circles): in the symmetry plane $z=0$ versus the Reynolds number; measurements in an other experiment ${ }^{1}$ (open circles), see discussion; (b) measurements at $R e=100$ versus $z$.

the separation surface is subjected to shear-layer instabilities above a critical Reynolds number $(R c=313$ for $h=10 \mathrm{~mm}$ and $R c=256$ for $h=5 \mathrm{~mm}$ ). The present study is concerned by the stationary regime only.

\subsection{Recirculation length measurements}

The recirculation length is obtained from the PIV measurements (as displayed in Fig. 3) by measuring the distance between the step corner and the point of reattachment on the bottom wall. This point is characterized by a zero longitudinal velocity in the extreme vicinity of the bottom wall. Fig. 4(a) represents the recirculation length in the plane $z=0$ obtained with the $10 \mathrm{~mm}$ high step versus the Reynolds number. It increases almost linearly in the stationary regime.

In order to characterize the topology of the separated zone, we perform several measurements of the reattachment length in the spanwise direction (corresponding to different PIV planes $z=\mathrm{cst}$ ). The recirculation length versus the spanwise coordinate $z$ is plotted in Fig. 4(b). These measurements made at $R e=100$ indicate a strong wall effect since the recirculation length ranges from $1.5 \mathrm{~h}$ to $7 \mathrm{~h}$. On both sides of the channel $(z<-70 \mathrm{~mm}$ and $z>70 \mathrm{~mm})$, we observe a side-wall effect similar to the one described by Armaly et al. [1] and later called wall-jets by Williams and Baker [6].

\footnotetext{
${ }^{1}$ Data from Armaly et al. [1]. The data have been rescaled using our definition of the Reynolds number.
} 
The striking observation concerns the centre part of the channel $(-50 \mathrm{~mm}<z<50 \mathrm{~mm})$, where we can see a spanwise modulation of the recirculation length with a wavelength of about $\lambda \sim 30 \mathrm{~mm}$.

\subsection{Transversal visualizations}

We perform the same kind of visualizations as those described in Section 3.1 but in transversal planes $(x=$ cst $)$.

A clear periodic spanwise structure of the flow is observable for both step height (Fig. 5(a) and (b)). The dye separates into five patches for the small step and into five mushroom-like patterns for the big step. These structures reveal the presence of counter-rotating longitudinal vortices. We observed this kind of spanwise structures for lower and higher Reynolds numbers in a range of 20 to 200. We never observed any threshold for this phenomenon in this range. Actually, these structures always appear after a very long time (typically 30 minutes) compared to the advection time of the dye passing above the separated region (1 minute). The disturbance of the velocity field induced by the longitudinal structures should then be very small compared to the basic flow.

The same spanwise wavelength is found for both step heights (Fig. 5 (a) or (b)) and is about $30 \mathrm{~mm}$. It corresponds to the spanwise wavelength observed in Fig. 4(b) for the reattachment length. We then find that the wavelength does not depend on the step height. Moreover, whatever the Reynolds number and for a given step height, we observe the same wavelength.

We checked the influence of the upstream ramp (with no step) on the flow using the experimental configuration described in Fig. 1(c). The picture in Fig. 5(c) displays the typical visualization we can observe. The dye remains homogenously distributed at the bottom wall. Sometime, a little cusp appears (as displayed in Fig. 5(c)) and disappears after a typical time of 10 minutes, but never any spanwise periodicities are observable.

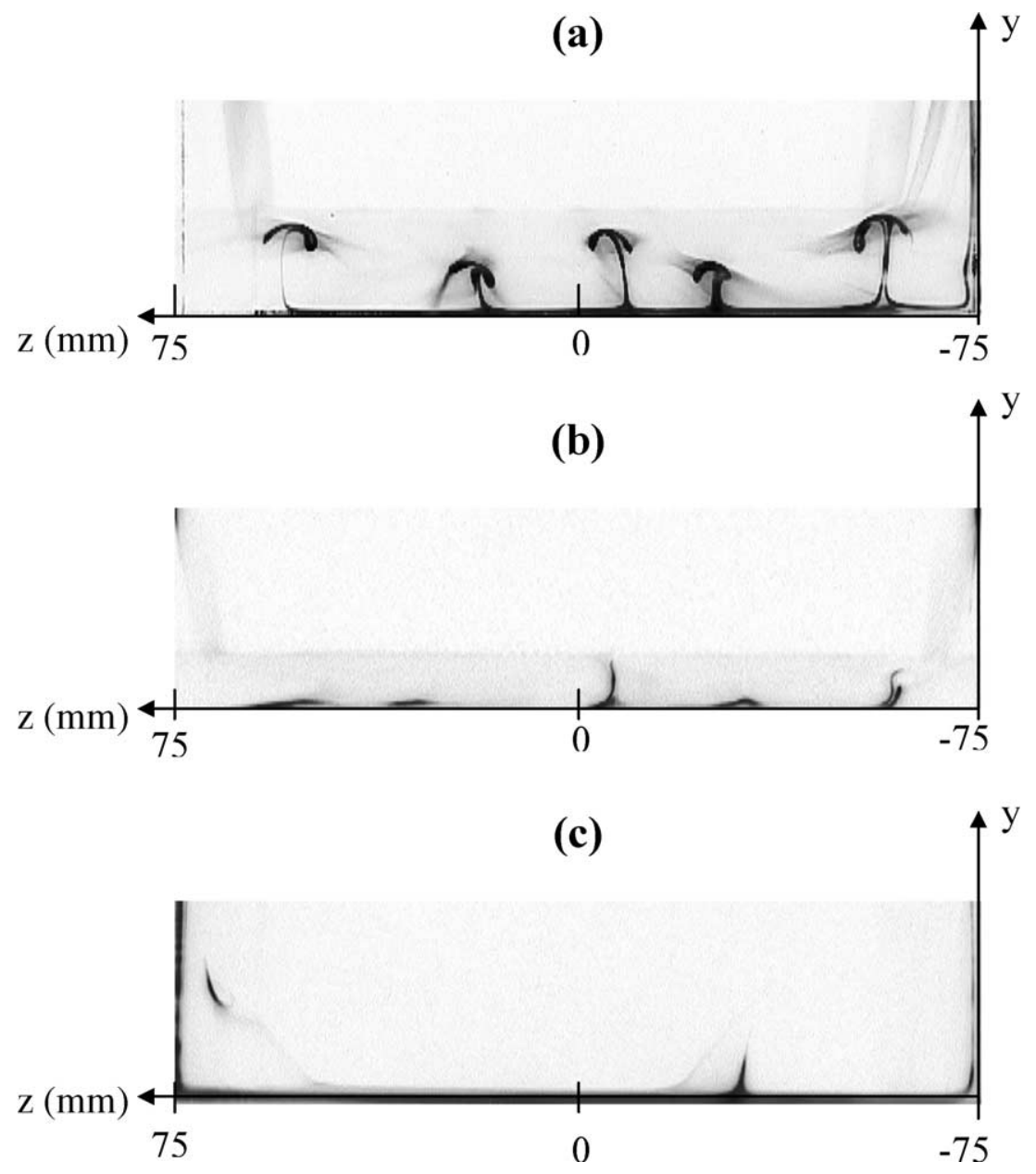

Fig. 5. Visualization of the flow in the plane $x=25 h$ at $R e=100$, the flow is coming in the direction of observation: (a) with the $10 \mathrm{~mm}$ high step; (b) with the $5 \mathrm{~mm}$ high step; (c) with the upstream ramp (no step). On the first two pictures we can see the step edge upstream. 
In the next part of the article, we propose a possible mechanism for the origin of the three-dimensional structure of the flow. Our strategy is to characterize the stability of a two-dimensional flow obtained by direct numerical simulation.

\section{Numerical simulation}

When there are curved streamlines in two-dimensional flow, three-dimensional instability may occur in the form of counterrotating vortices in the direction of the flow: it is called centrifugal instability. The necessary condition for the existence of this instability is given by the inviscid Rayleigh criterion which consists in considering the sign of a function $\Phi$ called the Rayleigh discriminant and computed from the two-dimensional basic flow.

\subsection{Numerical simulation}

So, our aim is not to reproduce the three-dimensional instability but to obtain the two-dimensional basic flow on which a centrifugal stability criterion will be applied in the following subsections.

The numerical domain is defined in Fig. 6(a). It represents exactly the longitudinal section of the hydrodynamic channel with the step of height $h=10 \mathrm{~mm}$. In particular, a special care is done to have a long enough downstream section to solve completely the recirculation region and to prevent numerical effect due to the outflow condition.

The boundary conditions are no slip wall conditions on the upper and lower part of the domain. The inflow condition is a flat velocity profile with the $U_{0}$ velocity so that the boundary layer grows before reaching the edge of the step. We impose an outflow condition at the exit of the domain.

We use a structured mesh with a very fine grid so that it can be used for a rather wide range of Reynolds number. The mesh is refined in the boundary layer regions, in the separation region, and in the recirculation bubble (Fig. 6(b)). The smallest resolution in the vertical direction is $0.25 \mathrm{~mm}$. The total grid size is 43000 cells.

As the Reynolds numbers are moderate, we perform direct numerical simulation DNS of the flow. The numerical procedure is based on a control volume, finite difference method. The equations are solved using the SIMPLE (Semi Implicit Method for Pressure Linked Equation) algorithm with an iterative line-by-line matrix solver.

\subsection{Rayleigh discriminant computation}

The application of the Rayleigh criterion (Rayleigh [10], Drazin and Reid [11]) gives a necessary (but not sufficient) condition of instability, and we will discuss the stabilizing effect of the viscosity in the following section.

The centrifugal instabilities can appear in a basic flow where the highest velocities are close to the centre of curvature of the streamlines. This situation corresponds to an algebraic radius of curvature opposite to the vorticity (the radius of curvature is

(a)

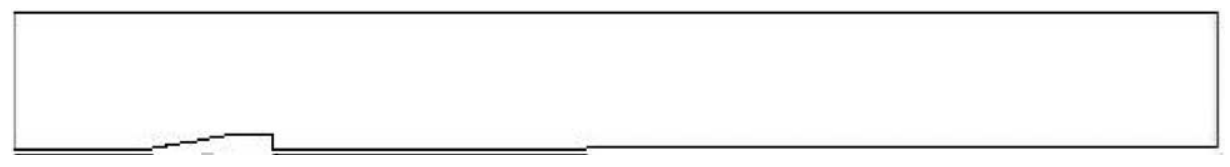

(b)

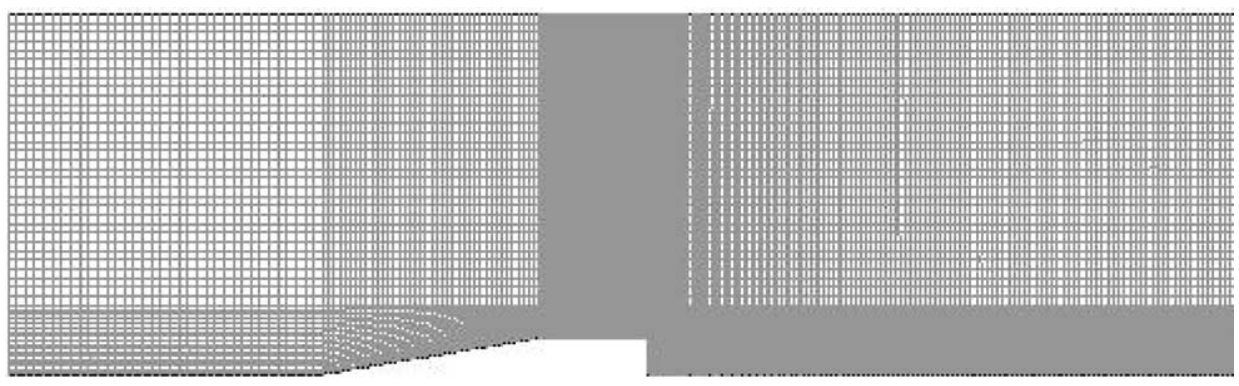

Fig. 6. (a) Numerical domain used for the computations representing the exact geometry of the experimental set-up; (b) numerical grid used for the computations (43000 cells). The grid is refined in the boundary layer regions and in the separation region. 


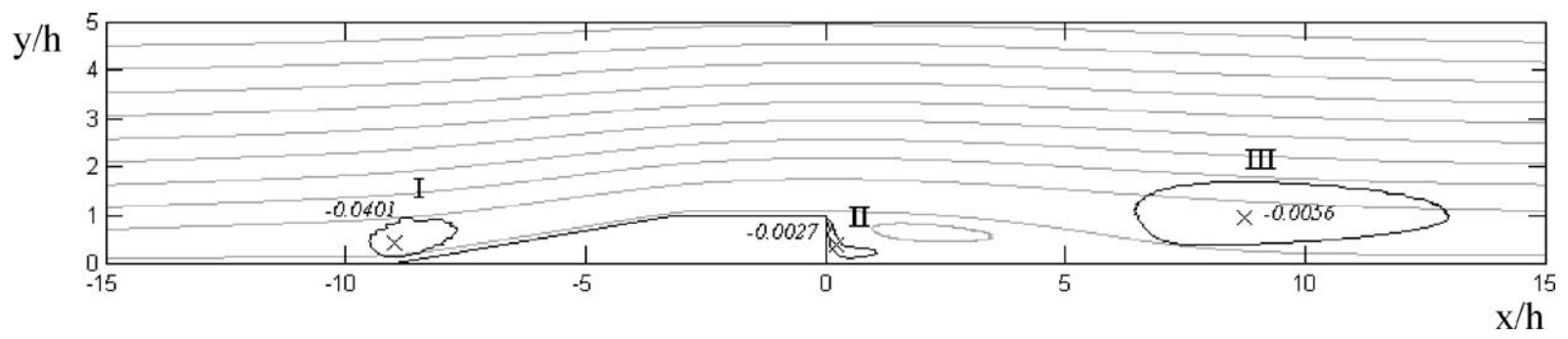

Fig. 7. Contour plot of the Rayleigh discriminant (black lines) superimposed with the streamlines obtained from the numerical simulation (grey lines) at $R e=100$. Three potentially unstable regions appear: each minimum is displayed with a cross and the contour plot around corresponds to its half-minimum value.

positive if the flow is locally counter-clockwise and negative if not). The following function $\Phi$ (called the Rayleigh discriminant) is computed numerically from the results of the $2 \mathrm{D}$ numerical simulation, its expression is as follows:

$$
\Phi(x, y)=\frac{2 U \varpi}{R}
$$

where $U(x, y)$ is the modulus of the velocity, $\omega(x, y)$ is the vorticity and $R(x, y)$ is the local algebraic radius of curvature. We computed this generalized Rayleigh discriminant, corresponding to a local criterion for a potential centrifugal instability (Mutabazi et al. [12,13], Sipp and Jacquin [14]). The local radius of curvature is computed following Sipp and Jacquin [14]:

$$
R(x, y)=\frac{U^{3}}{u a_{y}-v a_{x}},
$$

where $(u, v)$ are the components of the velocity field and $\left(a_{x}, a_{y}\right)$ the components of the convective acceleration $(\mathbf{u} \cdot \nabla) \mathbf{u}$.

The application of the Rayleigh criterion consists in considering the sign of $\Phi$ : when it is negative, the flow at the point $(x, y)$ is potentially unstable. The results of the computation are plotted in Fig. 7. We can distinguish three regions corresponding to three locations of high curvatures in the flow: the region in the front of the ramp I, the region in the recirculation zone II and finally the region just above the reattachment location III. The intensity of potential instability for each region is measured as the local minima of $\Phi$. It is -0.0056 in region III, -0.0027 in II and -0.0401 in region I. The region of potential instability is limited by the contour $\Phi=0$. However this contour is not well-defined because of the numerical noise. We then choose to estimate the spatial extension of each region as the contour plot at half the minimum. The hierarchy is different; the largest extension corresponds to region III, the intermediate to region I, the smallest to region II.

\subsection{Görtler number}

The Rayleigh criterion gives a necessary condition for the centrifugal instability but it does not take into account the stabilizing viscosity effect. The Görtler number [15] actually compares the curvature effects with the viscosity effects:

$$
G=\operatorname{Re}\left(\frac{\delta}{R}\right)^{1 / 2}=\frac{U \delta^{3 / 2}}{v R^{1 / 2}},
$$

where $R e$ is the Reynolds number based on $\delta$ (characteristic size of the unstable zone, which is the boundary layer thickness in the classical Görtler problem) and $v$ is the kinematic viscosity of the fluid. When the Görtler number is high enough (above a threshold that has to be defined by the stability analysis) the curvature effect dominates the viscosity effects and the flow is unstable.

With the numerical simulation we measure the local values of $U$ and $R$ at the three maximum locations of potential instability exhibited in Fig. 7. We define the characteristic size of each unstable zone $\delta$ as the width of the contour of the half-minimum value of the Rayleigh discriminant.

We perform several numerical simulations with the same grid from $R e=50$ to $R e=500$ (the resolution of the Navier-Stokes equations is maintained in the steady case). We first show in Fig. 8(a) the evolution of the recirculation length, which increases as the Reynolds number increases. In Fig. 8(b) we plot the local Görtler number defined in Eq. (3) of the three regions. We observe that the largest Görtler number is not found in region I where the modulus of the Rayleigh discriminant is the largest, but in region III. Moreover, in region I, the Görtler number saturates around 75 while it still increases in region III up to 400. In region II, the Görtler number remains, in comparison, very small and never exceeds 15. 

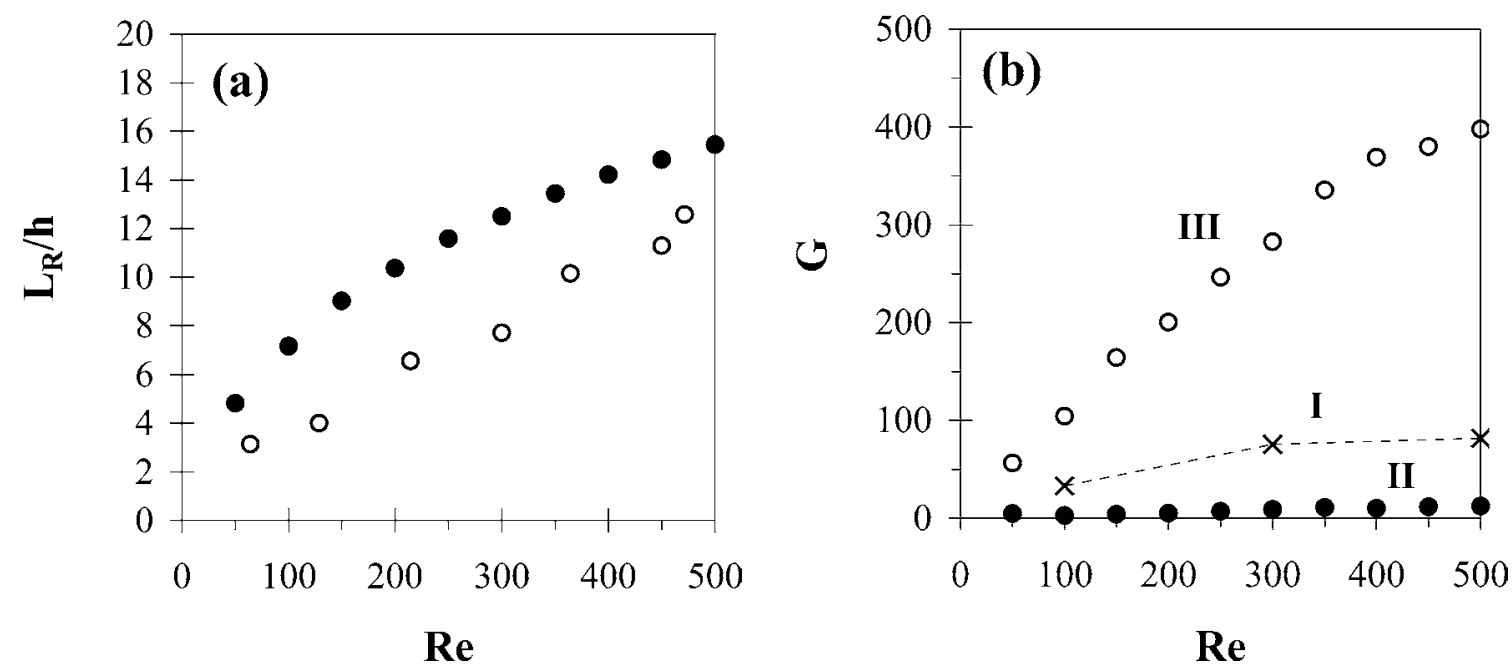

Fig. 8. Results of the 2D steady numerical simulation: (a) non-dimensional recirculation length (filled circles) versus the Reynolds number; measurements in an other experiment, ${ }^{2}$ see discussion; (b) Görtler number for each potentially unstable region versus the Reynolds number (crosses for region I, filled circles for region II and empty circles for region III).

\section{Discussion}

We first discuss the possibility of a centrifugal instability as the origin of the observed three-dimensional flow. Then, we compare our results to previous experimental and numerical results.

In order to understand the origin of the three-dimensionality in the experiment, we compute the Rayleigh discriminant of the two-dimensional basic flow. We find the basic flow to be potentially unstable in three regions (Fig. 7). However, to take into account the stabilization due to the viscosity, we compute a Görtler number for each regions (Fig. 8(b)). A relevant fact is that we do not observe any instability in our experiment due to the ramp alone (see Fig. 5(c)), we can then deduce that region I is stabilized by the viscosity. Consequently, the value of the Görtler number in region I is below the threshold of stability. It implies that region II should be stable since the Görtler number is always smaller in region II than in region I. On the other hand, the Görtler number of region III is always larger than the Görtler number of region I: it is then plausible that region III could be unstable through centrifugal instability.

In their recent numerical simulation, Barkley et al. [7] performs a linear stability analysis and show that a three-dimensional instability occurs in region II and not in region III. However, they do not give more indication about the mechanism of the instability they observe. At the moment, we do not understand this contradiction and the discussion about the three-dimensional instability origin is still open.

We now turn to other previous works [1-3,6]. Actually, our experiment is the first one to show a spanwise periodicity of the flow. Previous works $[1,6]$ report side-wall effects but not any intrinsic three-dimensional instability. Hence, it is very consistent that the experimental velocity field of $[1,6]$ in the symmetry plane of the step is similar to the result of the twodimensional simulation. In our case, the experimental flow is three-dimensional, and we do not retrieve the main characteristic of the numerical flow (for $R e=100$ ); the recirculation length is about $4.5 h$ in the experiment whereas it is $7 h$ in the simulation. We should also mention that neither the recirculation length in our experiment (Fig. 4(a)) nor in our numerical simulation (Fig. 8(a)) is consistent with the data in reference [1]. This discrepancy could lie in the big difference in the expansion ratios. In our experiment it is close to 1 (which corresponds to a near semi-infinite medium, the only characteristic length is the step height) whereas it is often close to 2 [1-3,5] (for this configuration the flow is strongly confined since the inlet channel width is equal to the step height). Furthermore, it is also possible that the expansion ratio modifies the two-dimensional basic flow, affects the value of the generalized Rayleigh discriminant and then the potential for centrifugal instability. Moreover, our results proving the existence of three-dimensional structures are consistent with the observations of Albensoeder et al. [16] in the case of cavities since the same physical mechanism and results were obtained.

\footnotetext{
${ }^{2}$ Data from Armaly et al. [1]. The data have been rescaled using our definition of the Reynolds number.
} 


\section{Conclusion}

We report the existence of a three-dimensional stationary structure with a periodicity in the spanwise direction in the flow over a backward-facing step. With the support of direct two dimensional numerical simulation, we show that the origin of the instability is consistent with a centrifugal instability which appears in the vicinity of the reattached flow and outside the recirculation bubble. However, since such instability has not been seen in experiment with a lower expansion ratio, it is possible that its existence is conditioned by the expansion ratio.

\section{Acknowledgements}

The authors would like to thank Dwight Barkley for very fruitful discussions. We are grateful to Jean-Charles Boueilh for his help in the PIV measurements.

\section{References}

[1] B.F. Armaly, F. Durst, J.C.F. Pereira, B. Schönung, Experimental and theoretical investigation of backward-facing step flow, J. Fluid. Mech. 127 (1983) 473-496.

[2] L. Kaiktsis, G.E. Karniadakis, S.A. Orszag, Onset of the three-dimensionality, equilibria, and early transition in flow over a backwardfacing step, J. Fluid. Mech. 231 (1991) 501-528.

[3] L. Kaiktsis, G.E. Karniadakis, S.A. Orszag, Unsteadiness and convective instabilities in two-dimensional flow over a backward-facing step, J. Fluid. Mech. 321 (1996) 157-187.

[4] J. Kim, P. Moin, Application of a fractional-step method to incompressible Navier-Stokes equations, J. Comput. Phys. 59 (1985) $308-323$.

[5] M. Lesieur, P. Begou, E. Briand, A. Danet, F. Delcayre, J.L. Aider, Coherent vortex dynamics in large-eddy simulations of turbulence, J. Turbulence 4 (2003) 016.

[6] P.T. Williams, A.J. Baker, Numerical simulations of laminar flow over a 3D backward-facing step, Int. J. Numer. Methods Fluids 24 (1997) 1159-1183.

[7] D. Barkley, M.G.M. Gomes, R.D. Henderson, Three-dimensional instability in flow over a backward-facing step, J. Fluid. Mech. 473 (2002) $167-190$.

[8] O. Cadot, S. Kumar, Experimental characterization of viscoelastic effects on two- and three-dimensional shear instabilities, J. Fluid. Mech. 416 (2000) 151-172.

[9] R.J. Adrian, Particle-imaging techniques for experimental fluid mechanics, Annu. Rev. Fluid Mech. 23 (1991) 261-304.

[10] J.W.S. Rayleigh, On the dynamics of revolving flows, Proc. Roy. Soc. London Ser. A 93 (1916) 148.

[11] P.G. Drazin, W.H. Reid, Hydrodynamic Stability, Cambridge University Press, 1981.

[12] I. Mutabazi, C. Normand, J.E. Wesfreid, Gap size effects on centrifugally and rotationally driven instability, Phys. Fluids A 4 (1992) 1199.

[13] I. Mutabazi, J.E. Wesfreid, Coriolis force and centrifugal force induced flow instabilities, in: E. Tirapegui, W. Zeller (Eds.), Instabilities and Nonequilibrium Structures IV, Kluwer Academic, 1993, pp. 301-316.

[14] D. Sipp, L. Jacquin, A criterion of centrifugal instabilities in rotating systems, in: A. Maurel, P. Petitjeans (Eds.), Vortex Structure and Dynamics, Springer, 2000, pp. 299-308.

[15] H. Görtler, On the three-dimensional instability of laminar boundary layers on concave walls, NACA Technical Memorandum $1375,1954$.

[16] S. Albensoeder, H.C. Kuhlmann, H.J. Rath, Three-dimensional centrifugal-flow instabilities in the lid-driven-cavity problem, Phys. Fluids 13 (1) (2001) 121-135. 